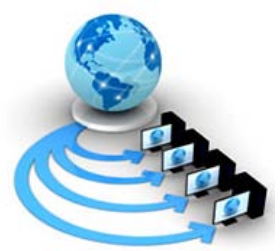

Volume 8, No. 7, July - August 2017

International Journal of Advanced Research in Computer Science

RESEARCH PAPER

\title{
IMPLEMENTATION OF NETWORK ARCHITECTURE, ITS SECURITY AND PERFORMANCE ANALYSIS OF VLAN
}

\author{
Syed Ehtesham Ul Haq \\ Department-Computer Science \\ Jamia Hamdard University \\ New Delhi
}

\author{
Suraiyaa Parveen \\ Department-Computer Science \\ Jamia Hamdard University \\ New Delhi
}

\begin{abstract}
The architecture of a computer network has developed with advances in technology. The design of secure computer network architecture to protect the integrity of information exchange is pursued by the commercial and financial sectors and at all levels of government agencies. Dynamic network speak to another way to deal with system architecture. It provides a much more flexible network infrastructure. The network security is mainly based on the network architecture. The purpose of this paper is to provide a broad survey on security in network system architecture.The first goal is to discuss various network architecture. The second goal is to highlight security issues in network architecture. Thus an inclusive presentation of network architecture, security issues is given.
\end{abstract}

Keywords: Peer - to - Peer Network, Client - Server Model, Network Security, authentication,VLAN

\section{INTRODUCTION}

Organizations are increasingly driven toward integrated interaction throughout their value chain: the ability to buy and sell using seamless electronic technologies is changing from a competitive advantage to a pre-requisite in many sectors. Current systems and communications standards are reducing the distinction between Local Area Networking and the remote operation of information systems using third-party telecommunications services. It is therefore becoming more realistic for smaller companies to connect their systems with those of their trading partners irrespective of organization size, structure, computing platforms or geographic location. [1]. Grounds network is basic and it assumes a critical part for any association.. Network architecture and its security are as important as air, water, food, and shelter. Computer network arrange security risk and system architecture are constantly significant issues. A campus network is an autonomous network under the control of a university which is within a local geographical place and sometimes it may be a metropolitan area network [2].

Orchestrate architecture is the arrangement of a correspondence network.It is a system for the determination of a system's physical segments and their utilitarian association and design, its operational standards and techniques, and in addition information groups utilize.. This concept was brought by Shreyas Dhakal In telecommunication, the specification of a network architecture may also include a detailed description of products and services delivered via a communications network, as well as detailed rate and billing structures under which services are compensated.[2] A layer is a collection of similar functions that provide services to the layer above it and receives services from the layer below it. On each layer, an example gives administrations to the cases at the layer above and demands benefit from the layer.[5]

The physical layer characterizes the electrical and physical details for devices.in specific, it characterizes the connection between a gadget and a transmission medium, for example, a copper or optical cable.This incorporates the format of pins,voltages, link determinations, centers,

\section{[fig 1 [11] ]}

repeaters, organize connectors, have transport connectors (HBA utilized as a part of capacity region arranges) The information interface layer gives the useful and procedural intends to exchange information between system substances and to recognize and perhaps rectify mistakes that may happen in the physical layer. Initially, this layer was planned for indicate and point multipoint media, normal for wide zone media in the phone framework. Neighborhood design, which included communicate proficient multi-get to media, was created autonomously of the ISO work in IEEE Project 802. IEEE work expected sub layering and administration capacities not required for WAN utilize. The vehicle layer gives straightforward exchange of information between end clients, giving dependable information exchange administrations to the upper layers. The vehicle layer controls the unwavering quality of a given connection through stream control, division/desegmentation, and mistake control. A few conventions are state and association arranged. [3]This implies that the vehicle layer can monitor the portions and re-transmit those that come up short. session layer gives a UI to the system where the client consults to set up an association. The client must give the remote deliver to be contacted.The operation of setting up a session between twprocesses is known as "binding".The introduction layer builds up setting between elements on the application layer, in which the 
higher-layer elements may utilize diverse language structure and semantics if the introduction benefit gives a mapping between them. On the off chance that a mapping is accessible, introduction benefit information units are exemplified into session convention information units, and gone down the stack The application layer is the OSI layer nearest to the end client, which implies that both the OSI application layer and the client collaborate straightforwardly with the product application. [6]This layer associates with programming applications that execute an imparting segment. Such application programs fall outside the extent of the OSI demonstrate organizing and steering 1-No single systems administration innovation can fulfill all requirements.Universal interconnection is desired.2-Protocols permit correspondence between hubs without understanding basic mechanisms.3-Internetworking is the procedure by which a gathering of unique, heterogeneous systems can be connected to shape a solitary intelligent network.4-The Internet is quite recently such an accumulation

.Routing is the instrument used to exchange information between systems to come to the right destination.Routing happens at the IP layer: switches don't know about transport and application layers.[4]

\section{[fig 2.1][11]}

Static routing:- In this sort of routing, manager is in charge of arrangement of all interfaces or course physically. Static routing is frequently used to help exchange routing data starting with one routing convention then onto the next (routing redistribution). Static routing can be utilized for little networks that require just a single or two courses. This is regularly more productive since a connection is not being squandered by trading dynamic routing data. Static routing can be utilized to characterize a leave point from a switch when no different courses are accessible or necessaryLayers can offer association arranged or connectionless administrations. Association arranged like phone system.Connectionless like postal system.Each benefit has a related Quality-ofadministration (e.g. dependable or inconsistent.

Default routing:- When executive don't have the thought regarding the goal arrange for this situation we utilize default routing. Executive must have the data of next hop.

Dynamic Routing:- in this sort of steering ,directing conventions are dependable to locate the best course consequently as indicated by their properties. the capacity of a framework, through which courses are described by their goal, to adjust the way that the course takes through the framework in light of a change in conditions.[5].[The adjustment is proposed to permit whatever number courses as would be prudent to stay substantial (that is, have goals that can be come to) because of the change. Frameworks that don't execute dynamic steering are depicted as utilizing static directing, where courses through a system are portrayed by settled ways (statically). A change, for example, the departure of a hub, or loss of an association between hubs, is not made up for. This implies anything that desires to take an influenced way will either need to sit tight for the inability to be repaired.[4]

\section{ROUTING PROTOCOLS:-1-RIP (Routing Information} Protocol) Properties of RIP:- It bolster class full system ,it can't bolster VLSM/CIDR,it AD value is 120,It backings most extreme fifteen hop count.,it gives intermittent update,It doesn't bolster multicast address,it doesn't bolster classless system [4]

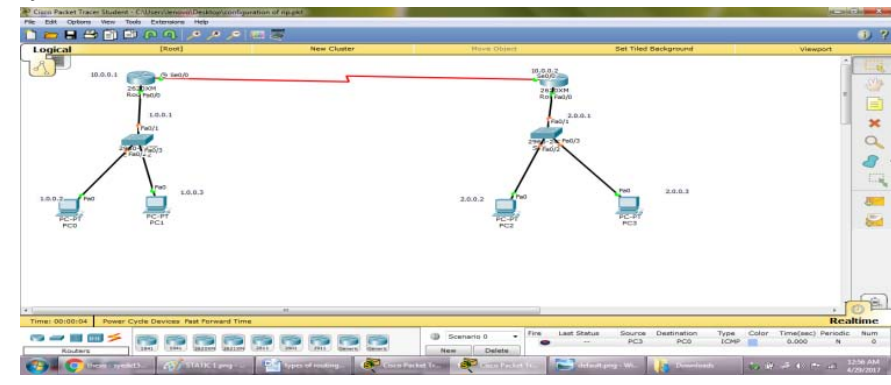

[fig 2]

2- RIP VERSION2 Properties of RIP V2:- It bolster class full/classless network.,it underpins multicasting (224.0.0.9),It supports bolsters VLSM/CIDR.,It gives incremental update.,It $\mathrm{AD}$ value is 120 and it bolster most extreme 15 hop count.,It is a separation vector convention. [4]

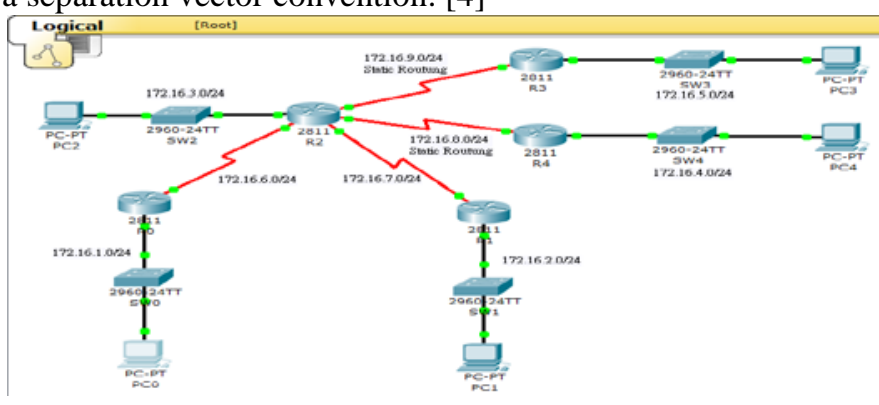

[fig 3][11]

3-EIGRP (Enhance Gateway Routing Protocol) Properties:It underpins VLSM/CIDR.,It bolsters class less/class full network,it underpins greatest 255 hops.,EIGRP multicast address is 224.0.0.10,AD value is 90.,Convergence is fast,the refresh clock is $5 \mathrm{~s}[4$

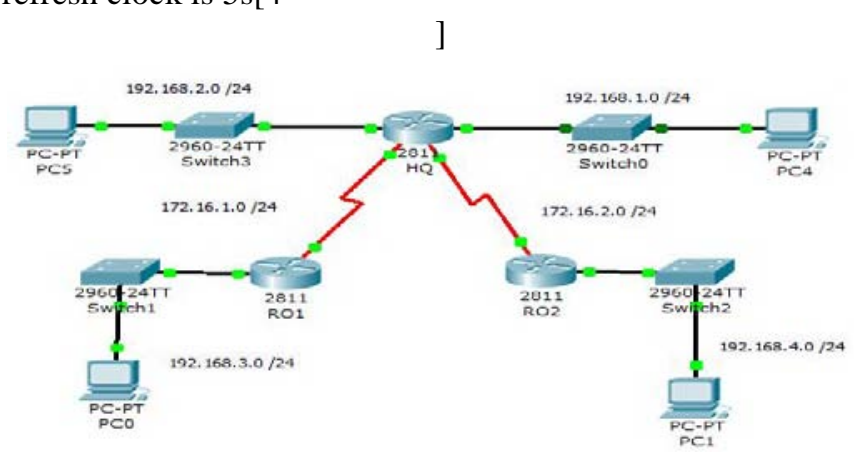

[fig 4][11]]

4-OSPF (Open Shortest Path First),it is additionally an open standard convention and it partitions in three categories,OSPF 
Neighbor table (neighbor discovery),OSPF Database Exchange (topology table), Route calculation.[4]

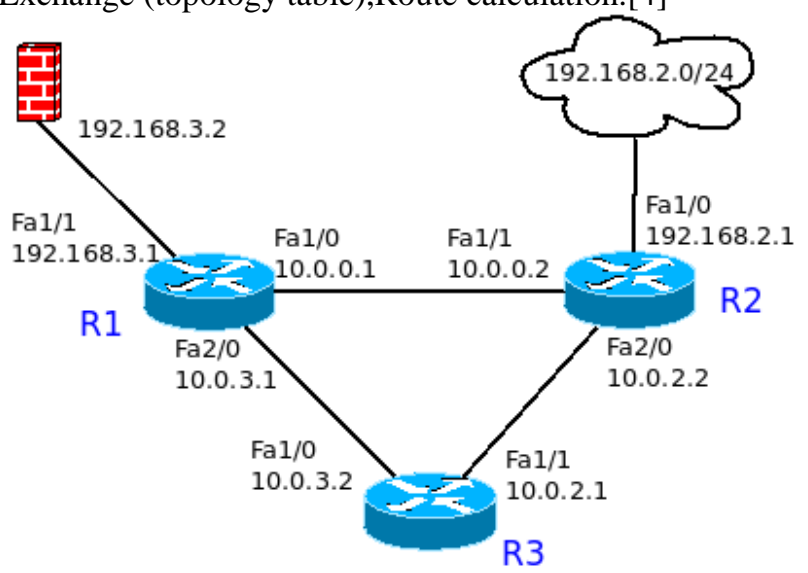

[fig 5 [11]

\section{EXPERIMENTAL SECTION}

\section{VLAN Architecture}

All the above issues, and significantly more, can be overlooked with the making of VLANs. As we probably am aware to make VLANs, you require a layer 2 switch that backings such convention. Many people new to the systems administration field bring the misguided judgment that it's a matter of just introducing extra programming on the customers or change, keeping in mind the end goal to "empower" VLANs all through the system - this is absolutely mistaken. It is never valid, rather we have VLAN empowered switches like cisco impetuses switches for the cisco system.[7] VLANs include a large number of numerical computations, they require uncommon equipment which is incorporated with the switch and your switch should hence bolster VLANs at the season of procurement, else you can't make VLANs on it. Each VLAN made on a switch is a different network.[8] This implies a different communicate area is made for each VLAN. Organize communicates, as a matter of course, are separated from all ports on a switch that are not individuals from the same VLAN and this is the reason VLANs are vital in today's substantial system like Ebonyi State University arrange as they help segregate organize fragments between each segment.

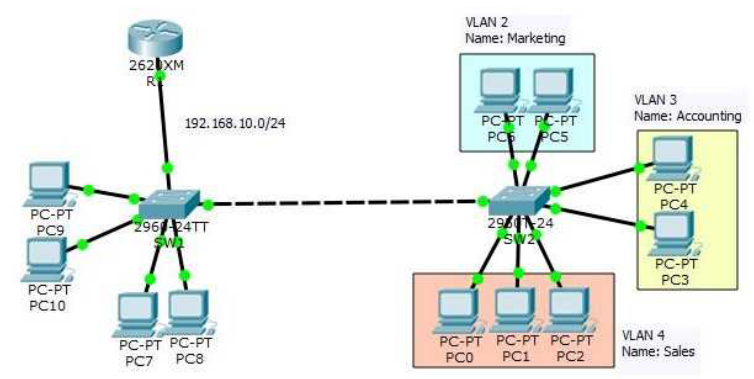

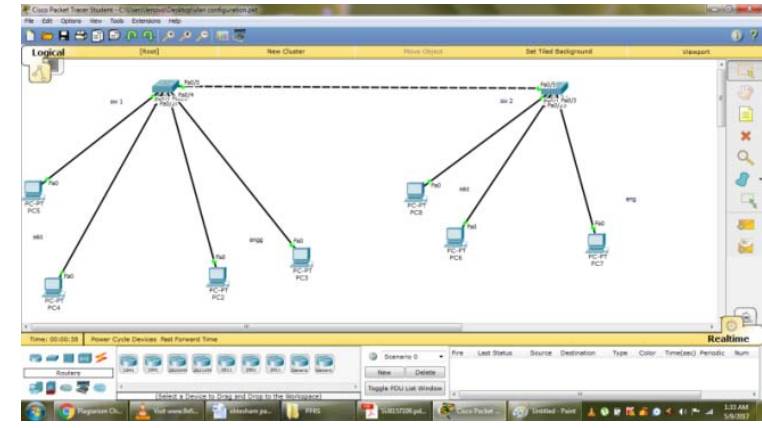

[fig 6 [11]]

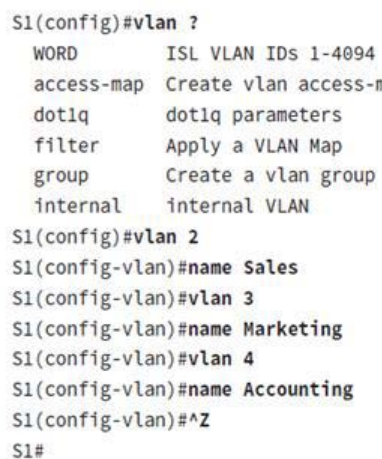

Creating VLAN in CLI (source: CCNA Routing and Switching, 2013)

\section{Configuration of switch:-}

switch (config) \#line console zero(0), \#password CISCO \#login $\downarrow$

\section{Configuration of VTY}

switch (config)\#line VTY 0 \#password CISCO \#login $\downarrow$

switch (config)\#enable secret password $\downarrow$

To check VLAN IP:-

\# Show ip int brief.

To check MAC table:-

\#MAC -address-table $\downarrow$

How to set an ip:-

switch(config)\#int vlan $1 \downarrow$

\# ip address 1.0.0.0 subnet mask $\downarrow$ \#no shut $\downarrow$

\#do wr.

\section{CREATION OF VLAN:-}

To check VLAN

\#show VLAN

To create vlan

Config\#VLAN2

\# name accounts

\#VLAN 3

\#nameHR

HOW TO GIVE MEMBERSHIP

config \#VLAN2

\# name accounts

\# int fa $0 / 1$.

\#switchport access VLAN 


\section{TYPES OF LINK}

i)Access link:- All port of impetus switch be default is get to interface. Get to connection can convey information in single VLAN.[6]

ii)Trunk link:- It can convey numerous VLAN information at an opportunity to make a trunk connect. Interface ought to be quick Ethernet.[6]

\section{HOW TO CREATE TRUNK LINK}

switch (config) \#int fa o/1」 \#switchport mode trunk \#do wr

\section{HOW TO CHECK TRUNK}

\#show int trunk

\section{ENTER VLAN ROUTING:-}

With the help of inter VLAN routing we can make communication between different we can make communication different different VLAN

router (config)\#int fa $0 / 0\lrcorner$

$$
\text { \#no client }
$$

\#encapsulation dot $1 \mathrm{Q} 1 \rightarrow$ VLAN id $\lrcorner$

\#ip address 10.0.0.1 255.0.0.0

\#no short $\lrcorner$

\#no wr.

\#int fa $0 / 0.2$.

\#encapsulation dot 1Q 2.

\#ip address 20.0.0.1 255.0.0.1」

\#no short $\downarrow$

\#int fa $0 / 1\lrcorner$

\#encapaulation30.0.1 255.0.0.0.

\#ip address 30.0.0.1 255.0.0.0

\#no client

\#do wr

A flat network is a PC organize configuration approach that plans to lessen taken a toll regarding upkeep and organization. Flat networks are intended to decrease the quantity of switches and switches on a PC arrange by associating the gadgets to a solitary switch rather than isolated switches, or by utilizing system center points as opposed to changes to interface gadgets to each other. The topology of a flat network is not sectioned or isolated into various communicate ranges by utilizing switches and switches. Not at all like Virtual Local Area Network plan, the system is not consistently isolated into various communicate spaces. By and large, all gadgets on the level system have a place with a similar communicate area.[10]

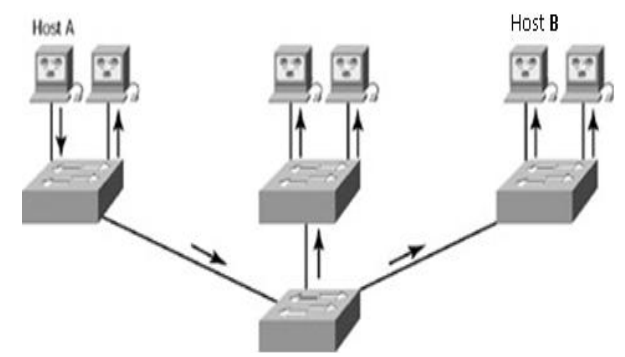

[fig 7]
Broadcast Flow on Flat Network structure (Source: Mega Guide, CCNA 640-802, www.preplogic.com)

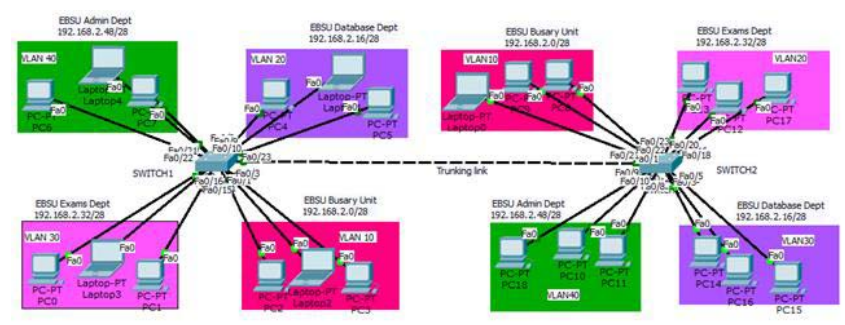

[fig 8][10]

BENEFITS OF VLAN:- Scalability: Arrange includes, moves, and changes are accomplished effortlessly by simply designing a port into the proper VLAN and allotting hosts to the same VLAN layer 2 switches just read outlines for sifting since they don't take a gander at the Network layer convention. In like manner we additionally realize that as a matter of course, changes forward communicates to all ports with the exception of the port that got the communicate to be forwarded.[7] But in the event that you make and execute VLANs, you're basically making littler communicate spaces at layer 2. Accordingly, communicates conveyed from a hub in one VLAN won't be sent to ports designed to have a place with an alternate VLAN. Be that as it may, on the off chance that we allocate switch ports or clients to VLAN bunches on a switch or on a gathering of associated switches, we pick up the adaptability to only include just the clients we need to let into that communicate space paying little heed to their physical location.[7] This setup can likewise work to square communicate storms brought on by a broken system interface card (NIC) and also keeping a middle gadget from proliferating communicate storms all through the whole internetwork. Another extraordinary favorable position is that as VLAN gets too huge, you can essentially make more VLANs to shield the communicates from devouring excessively transmission capacity. The less clients in a VLAN, the less clients

- Security: VLANs give improved system security. In a VLAN organize condition, with numerous communicate areas, arrange directors have control over each port and client. A pernicious client can no longer correspondence. A level internetwork's security used to be handled by associating center points and switches together with switches. So it was essentially the switch's business to look after security. This game plan was truly inadequate for a few reasons. Initially, anybody associating with the physical system could get to the system assets situated on that specific physical LAN. Furthermore, for anybody to watch all activity crossing that system is to just connect a system analyser to the center. What's more, like the last, an unnerving truth, is that clients could without much of a stretch join a workgroup by simply connecting their workstations to the current center. Yet, that is precisely what improves VLANs an innovation. In the event that you assemble and make various communicate social occasions, you can at present have mean control over each port and customer therefore restricting people from unapproved access by interfacing workstations to any switch port. VLANs can be made in congruity with a specific customer's necessity for the framework resources. [8]SWITCH1 and SWITCH 2 can be organized to light up a framework organization station 
about unapproved access to those fundamental system assets. Furthermore, in the event that you require between VLAN correspondence, you can actualize limitations on a switch to guarantee access of level of clients and place confinements on equipment locations, conventions, and simply connect their workstation to any switch port and sniff the system movement utilizing a parcel sniffer. [9]The organize manager controls each port and whatever assets it is permitted to utilize. VLANs help to confine touchy activity beginning from an undertaking division inside itself. Security issues are dependably the significant difficulties we confront in information

Creating Workgroups: A gathering of clients that need a strangely abnormal state of security can be put into its own VLAN so that clients outside of that VLAN can't speak with it. This suggests in an association every division can be made autonomous from different offices.

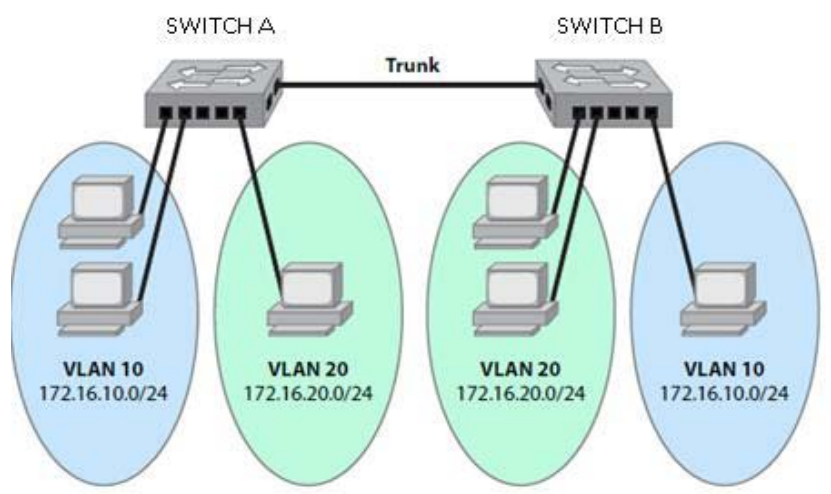

[fig 9] Trunking protocol between switches (Source: Mega Guide, CCNA 64 0-802, www.preplogic.com)

$\square$ Cost effective: Taken a toll investment funds can be seen by dispensing with the requirement for extra costly system gear like switches. VLANs will likewise enable the system to work all the more effectively and order better utilization of data transmission and assets. [8]

$\square$ Easy Troubleshooting: By gathering our system clients and assets into various VLANs, issues radiating in the system can without much of a stretch be distinguished and settled by negligible following gathering such has have a place to[9].

$\square$ Integrity: As a legitimate gathering of clients by capacity, VLANs can be viewed as autonomous from their physical or geographic areas. In this way, our University information can be dealt with without include. [7]

$\square$ Broadcast Control: The communicate of the system can be overseen and controlled by making numerous VLANs which constantly expands the quantity of communicate areas while diminishing their size. Communicates happen in all convention, however how frequently they happen relies on upon the sort of convention, the application(s) running on the internetwork, and how these administrations are utilized. Some more established applications have been changed to lessen their transmission capacity utilization, however there's another era of uses that are so data transfer capacity ravenous which expend each data transmission they see like mixed media applications.[10] As in the event that they weren't sufficient inconvenience, variables like defective hardware, insufficient division, and created firewalls can really bother the issues starting at now brought on by these impart heightened applications. The dominant part of this has new estimation to network arrange and presents a heap of new challenges for an administrator. Determinedly guaranteeing that framework is suitably segmented as showed up in above controls sort out impart from inducing all through the entire framework. [6]Therefore, a convey from EBSU Database Dept VLAN in can't be seen by EBSU Admin Dept, EBSU Bursary Unit and EBSU Exams Dept VLANs. Likewise, the best way to deal with do that is through key trading and controlling. Since changes have ended up being more sensible, a large number individuals had supplanted their level focus frameworks with flawless traded framework and VLAN conditions. All devices inside a VLAN are people from a comparative convey space and get all imparts huge to it. Normally, these conveys are filtered from all ports on a switch that aren't people from the same VLAN. Consequently, with VLAN use broadcast.[6]

\section{CONCLUSION}

Despite the fact that there are some numerous issues confronting network and information correspondence today however security has dependably been the most vital test. Each effort is dependably how to relieve frailty issues and guarantee that data is imparted to proper goals without trade off. This paper had sufficiently talked about the need to implement Virtual Local Area Network (VLAN). Passing by the standard level Local Area Network foundation where each clients have a place with one communicate space diverse arrangement of system frailties exist. On account of an effort arrange having basic record servers, application servers, hierarchical databases and other private data, this would imply that all clients would have square with get to benefits to these assets. To viably keep such circumstances from operational system we have to limit access at the network level by portioning the current network into various communicate spaces, consequently, the need of Virtual Local Area Network (VLAN). As opposed to ordinarily level LAN engineering where each hosts are associated without division; we break a huge communicate space into various sizes of communicate areas by making Virtual Local Area Networks (VLANs). This VLAN engineering which is a legitimate gathering of system clients and assets associated with officially characterized ports on a switch would be of enormous advantage as delineated in the work. On the whole, this work thoroughly $\mathrm{x}$-rayed the advantages of VLAN.

\section{ACKNOWLEDGMENT}

I would like to thank sincerely to the jamia hamdard university faculty of computer science and technology for the invaluable guidance, constant assistance ,support ,endurance and constructive suggestion.

\section{REGERENCES.}

[1]. Dr M.Lilly Florence, Dr D Swamydas "security issues in computer network architecture”volume2,no-7 july 2011

[2]. Andrew S. Tanenbaum,David J .Wetherale”computer networks"fifth edition, volume-5,2011,pearson publication $\operatorname{pp}(17-45)$

[3]. Oliver Bonventure "computer networking principles protocols and practices" third- edition ,vol-3,2011,pp-(25-228),saylor foundation publisher.

[4]. Al-Akhras, M.A.,’Wireless Network Security Implementation in Universities" In Proc. of Information and Communication Technologies,2006,ICTTA’06.,Vol.2,pp.3192-3197,2006 
[5]. Goers, William C., Brenner, Michael R., "Implementing a Management System Architecture", Bell Labs Technical Journal, Oct-Dec 2000, Vol. 5, Issue 4, p31-43

[6]. Milan Yu and Jennifer Rexford, Princeton University,Xin Sun and Sanjay Rao, Purdue University, Nick Feamster, Georgia institute of Technology. "A survey of Virtual LAN Usage in Campus Networks" IEEE Paper in IEEE Communications Magazine July, Volume: 49,2009, Issue: 7 pp. 98-103.

[7]. Yu-Wei Eric Sung, Sanjay G. Rao, Geoffrey G. Xie,David A. Maltz, Purdue University."Towards systematic Design of Enterprise network” Networking, IEEE/ACM Transactions, Volume: 19, 2011,Issue: 3 pp. 695-708.
[8]. Rajiv O. Verma, S.S. Shriramwar "Security Optimization of VTP Model in an Enterprise VLAN" International Journal of Electronics Communication and Computer Engineering Volume 4,2007, Issue 3, ISSN (Online): 2249-071X, ISSN (Print): 2278-4209.

[9]. Design and Implementation of a secure campus network volume 5, Issue-7 July, 2015,' Mohammad Nadir Bin Ali, Mohammad Imran Hussain, Mohammad Masud Parvez; 10-. UC Davis” VLAN information Network 21”,Vol.7 ,no. 2,nov(1998),it times[online].http://net21.ucdavis.edu/newvlan.html.

[10] -.www.goggle map.com 